\title{
Fabrication of Chitosan-complexed Electrode and Evaluation of Its Efficiency in Removal of Copper Ion from Aqueous Solution
}

\author{
Young-Chan Yoon ${ }^{1, \mathrm{a}}$, Kyung-Bae Kim ${ }^{1, \mathrm{~b}}$, Dong-Hee Lee ${ }^{1, \mathrm{c}}$, Chu-Sik Park ${ }^{2, \mathrm{~d}}$ and Young-Ho \\ $\mathrm{Kim}^{1, \mathrm{e}, *}$ \\ ${ }^{1}$ Department of Chemical Engineering and Applied Chemistry, Chungnam National University, 220 \\ Gung-dong, Yuseong-gu, Daejon 34134, Republic of Korea \\ ${ }^{2}$ Hydrogen Energy Research Center, Korea Institute of Energy Research, 71-2 Jang-dong, Yuseong-gu, \\ Daejeon 34129, Republic of Korea

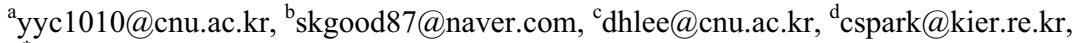 \\ e, ${ }^{*}$ yh_kim@cnu.ac.kr
}

\begin{abstract}
In this study, we fabricated chitosan/PVA/activated carbon complexed electrode to remove copper ion from aqueous solution. The prepared composite electrode was analyzed by BET and SEM to investigate its physicochemical properties. Electrochemical properties of prepared composite electrodes were analyzed via cyclic voltammetry. Adsorption performance of copper ion on chitosan composite complexed electrodes was evaluated. Almost similar pore size distribution results were observed in the series of ACP not included electrodes while observed differences in pore size distribution for the ACP included one. Cyclic voltammetry results exhibited that oxidation-reduction reaction does not occur in a potential range of $-1.0 \sim 1.0 \mathrm{~V}$. The amount of copper ion during adsroption reaction is increase according to increase of adsorption potential to $1.0 \mathrm{~V}$.

Keywords: Chitosan; PVA; Activated carbon; Electrode; Electrosorption
\end{abstract}

\section{Introduction}

Removal of copper ion from aqueous solution is an important issue because expose to high concentration of copper ion for a long period is harmful for human and other living being. Several technologies such as precipitation, ion exchange, electrode filtration, coagulation, flocculation, flotation and electrochemical treatment have been used to remove the copper ions in waste water [1-4].

Electrosorption is one of the promising considerable copper removal techniques because it has many advantages compare to previous technologies; for examples, low energy consumption, environmental friendliness, and simplified step. In detail, ions are extracted from aqueous solution by applying an electric potential between a pair of electrodes, in

*Corresponding author: yh_kim@cnu.ac.kr 
which the ions are temporarily stored via the formation of an electrical double-layer between the electrode and electrolyte interface [5]. Electrosorption can also improve the energy efficiency because the process operates at a low voltage region. Moreover, it is well known as eco-friendly process that does not produce the secondary by-product because it can proceed with desorption by applying a reverse potential in an aqueous solution [6].

Electrosorption efficiency is related to the specific surface area of electrode and it also affected by the type of surface functional group. Chitosan has a large amount of the amino group. It can form a chelate with copper ions in the solution using a lone pair of electrons and which can absorb the copper ions. Therefore, many studies have been conducted relating to the removal of copper ions using chitosan [7-9].

In this study, we prepared chitosan complexed electrode. It composed with chitosan and activated carbon, and polyvinyl alcohol used as a binder to removal of copper ions from the aqueous solution. Physical and chemical properties of the complexed electrode were analyzed by the BET and SEM, and its electrochemical properties were analyzed by cyclic voltammetry. Also, applied potential varied to evaluate its copper ion removal efficiency.

\section{Experimental}

\subsection{Fabrication of Chitosan-complexed Electrode}

$1 \mathrm{wt} \%$ acetic acid solution using deionized water and acetic acid (99.0\%, Deajung) and chitosan (medium molecular weight, Sigma-Aldrich) was mixed and stirred for $24 \mathrm{~h}$ at $50{ }^{\circ} \mathrm{C}$ to prepare chitosan solution. PVA solution was prepared by adding polyvinyl alcohol (PVA, Mw 31,000-50,000, 98-99\% hydrolyzed, Sigma-Aldrich) a weight ratio of 15 to deionized water. Afterwards, PVA solution stirred for $12 \mathrm{~h}$ at $70{ }^{\circ} \mathrm{C}$ in a water bath. Prepared chitosan solution and $15 \mathrm{wt} \%$ PVA solution was homogeneously mixed using a mechanical stirrer for $2 \mathrm{~h}$. The CTS/PVA mixture was coated on graphite sheet (150 $\mu \mathrm{m}$ of thickness, Dongbang Carbon Co.) using a doctor blade to a thickness of $450 \mu \mathrm{m}$. Afterwards, chitosan-complexed electrode was dried for $12 \mathrm{~h}$ at room temperature and baked at $100{ }^{\circ} \mathrm{C}$ for $30 \mathrm{~min}$ in an oven. Finally, it was soaked for $1 \mathrm{~h}$ in $1 \mathrm{M} \mathrm{NaOH}$ solution, and washed with deionized water until the $\mathrm{pH}$ of the washed liquid reached to that of deionized water.

Fabrication of chitosan-complexed electrode containing activated carbon powder (ACP, P-60, Deadong AC Corp., Specific surface area of $1,260 \mathrm{~m}^{2} / \mathrm{g}$ ) was similar to the procedure as stated above. For this, ACP was added into the PVA solution before mixing chitosan/PVA. The fabricated complexed electrode was named according to the ratio of chitosan, PVA and ACP. (Ex. CTS/PVA/ACP $=1 / 2 / 3$ for M123).

\subsection{Characterization of the Chitosan-complexed Electrode and Adsorption Experiment}

Physical and chemical properties of the prepared chitosan-complexed electrode were analyzed by SEM and BET. Electrochemical properties of the chitosan-complexed electrode were analyzed by cyclic voltammetry measurement. M311, M212 and M113 containing activated carbon were selected as cyclic voltammetry measurement samples. Fig. 1 shows the schematic diagram of the equipment for electrochemical experiment. The water analyzer (HS-3100, HUMAS) was used to measure the concentration of copper ions in aqueous solution. 


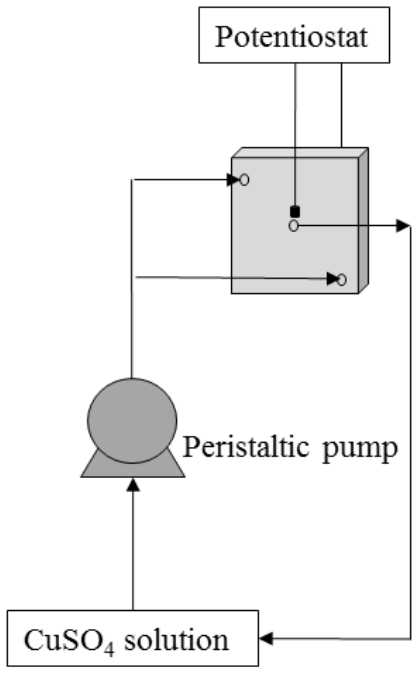

Fig. 1. Schematic diagram of copper ion electrosorption process by chitosan-complexed electrode.

\section{Results and Discussion}

\subsection{Physical and Chemical Characterization}

Fig. 2 shows the morphologies of CTS/PVA and CTS/PVA/ACP complexed electrodes. Two complexed electrode was observed smooth surface, and the cross sectional view was similar to each other. The roughness of surface with differ from CTS/PVA complexed electrode by adding of activated carbon. The $3 \mathrm{~d}$ network pores observed for the ACP added electrode in the cross section of the electrode.
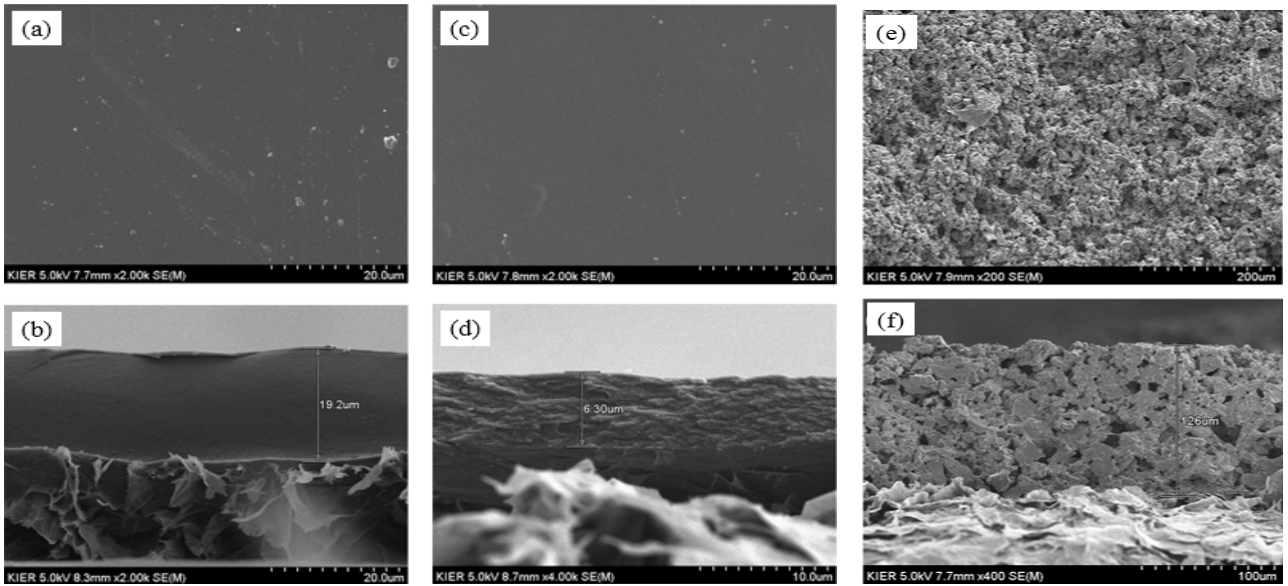

Fig. 2. SEM images of prepared CTS/PVA and CTS/PVA/ACP electrodes. Surface view (a) M710 , (c) M410(e) M113 and cross sectional view (b) M710, (d) M410 and (f) M113. 
Fig. 3 shows the pore size distribution results for the CTS/PVA complexed electrode and CTS/PVA/ACP complexed electrode calculated by the BJH method using the nitrogen adsorption-desorption isotherm result. Almost similar pore size distribution results were observed in the series of ACP not included electrodes while observed differences in pore size distribution for the ACP included one. This result indicates that the pore structure was affected by the addition of ACP.

(a)

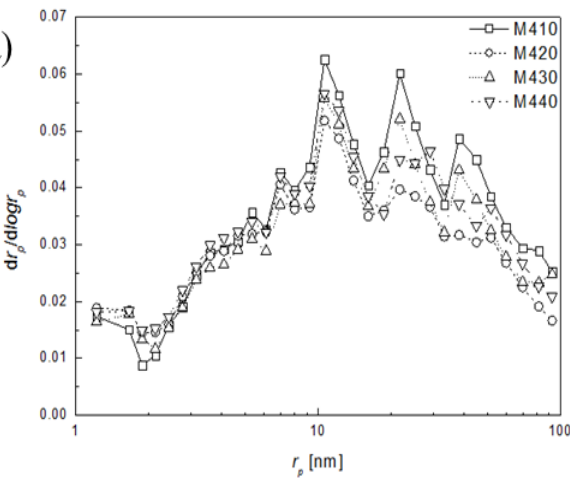

(b)

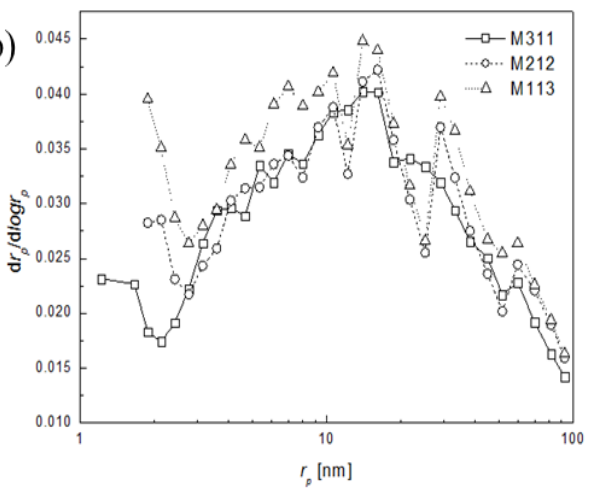

Fig. 3. Pore size distribution of fabricated (a) CTS/PVA and (b) CTS/ACP/PVA electrode.

\subsection{Electrochemical Characterization of Chitosan-complexed Electrode}

Fig. 4 shows the cyclic voltammetry of chitosan-complexed electrode was measured to analyze the electrochemical properties with the potential range is from $-1.0 \mathrm{~V}$ to $1.0 \mathrm{~V}$ and $0.01 \mathrm{mV} / \mathrm{s}$ scan rate under the presence of $20 \mathrm{ppm} \mathrm{CuSO}_{4}$ solution. The cyclic voltammogram did not have a prominent peak in a range of $-1.0 \sim 1.0 \mathrm{~V}$, which means that oxidation-reduction reaction does not occur in this experimental range.

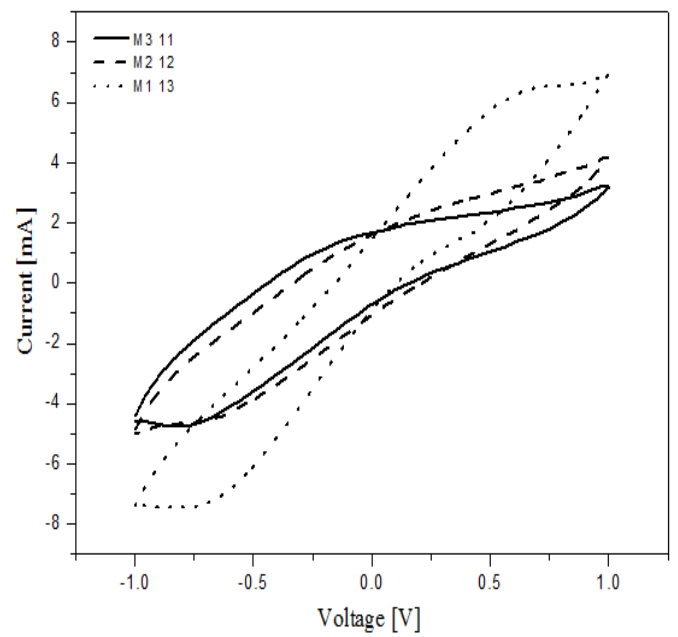

Fig. 4. Cyclic voltammograms of M311, M212 and M113 electrodes in copper ion 20 ppm solution; $-0.1 \sim 0.1 \mathrm{~V}$ of voltage range, $0.01 \mathrm{mV} / \mathrm{s}$ of scan rate. 
Fig. 5 shows the changes of copper ion concentration in the reservoir solution during adsorption reaction. In this case, CTS/PVA/ACP complexed electrode of M311 was used, and the adsorption potential varied from 0.2 to $1.2 \mathrm{~V}$. It confirmed that the copper ion adsorption equilibrium time using chitosan-complexed electrode in the electrochemical adsorption is around $300 \mathrm{~min}$. The adsorption amount of the copper ions kept constant irrespective of the change of potential. From the results, the amount of adsroption is increase according to increasing of applied adsorption potential, and amount of adsorption is similar at $1.0 \mathrm{~V}$ and $1.2 \mathrm{~V}$.

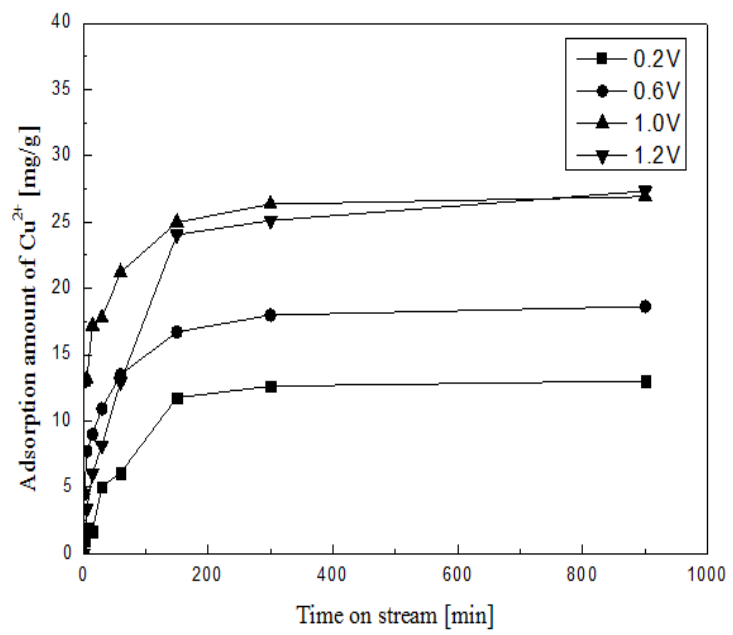

Fig. 5. Effects of adsorption voltage and adsorption time on copper ion electrosorption using M311 electrode.

\section{Conclusions}

We successfully fabricated chitosan-complexed electrode with a different composition of chitosan, PVA and activated carbon for removal of copper ions in the aqueous solution. Physical, chemical and electrochemical properties of fabricated electrodes were analyzed. The specific surface area of chitosan-complexed electrode was increased by adding of activated carbon, which confirmed by SEM and BET. Electrosorption result indicates that the equilibrium was reached in $300 \mathrm{~min}$ irrespective of the change of the adsorption potential. The amount of copper ion during adsroption reaction is increase according to increase of adsorption potential to $1.0 \mathrm{~V}$. Cyclic voltammetry results exhibited that oxidation-reduction reaction does not occur in a potential range of $-1.0 \sim 1.0 \mathrm{~V}$.

\section{Acknowledgements}

This research was supported by Basic Science Research Program through the National Research Foundation of Korea(NRF) funded by the Ministry of Science, ICT \& Future Planning (NRF-2015R1A2A2A01002828). 


\section{References}

1. M. Bhagat, J.E. Burgess, A.P.M. Antunes, C.G. Whiteley, J.R. Duncan, Precipitation of mixed metal residues from wastewater utilising biogenic sulphide, Minerals Engineering 17 (2004) 925-932.

2. A. Stajčić, A. Nastasović, J. Stajić-Trošić, J. Marković, A. Onjia, F. Radovanović, Novel membrane-supported hydrogel for removal of heavy metals, J. Environ. Chem. Eng. 3 (2015) 453-461.

3. Y.J. Liang, L.Y. Chai, X.B. Min, C.J. Tang, H.J. Zhang, Y. Ke, X.D. Xie, Hydrothermal sulfidation and floatation treatment of heavy-metal-containing sludge for recovery and stabilization, J. Hazard. Mater. 217 (2012) 307-314.

4. M. Prica, S. Adamovic, B. Dalmacija, L. Rajic, J. Trickovic, S. Rapajic, M. Becelic-Tomin, The electrocoagulation/flotation study: The removal of heavy metals from the waste fountain solution, Process Saf. Environ. Prot. 94 (2015) 262-273.

5. S.Y. Huang, C.S. Fan, C.H. Hou, Electro-enhanced removal of copper ions from aqueous solutions by capacitive deionization, J. Hazard. Mater. 278 (2014) 8-15.

6. Y. Han, X. Quan, S. Chen, S. Wang, \& Y. Zhang, Electrochemical enhancement of adsorption capacity of activated carbon fibers and their surface physicochemical characterizations, Electrochim. Acta. 52 (2007) 3075-3081.

7. O.A. Monteiro, C. Airoldi, Some thermodynamic data on copper-chitin and copper-chitosan biopolymer interactions, J. Colloid Interface Sci. 212 (1999) 212-219.

8. E. Guibal, Interactions of metal ions with chitosan-based sorbents: a review, Sep. Purif. Techno. 38 (2004) 43-74.

9. M.W. Wan, C.C. Kan, B.D. Rogel, M.L.P. Dalida, Adsorption of copper (II) and lead (II) ions from aqueous solution on chitosan-coated sand, Carbohydr. Polym. 80 (2010) 891-899. 